\title{
Comparative Study between Validity Indices to Obtain the Optimal Cluster
}

\author{
Hanifi Meroufel*, Habib Mahi, Nezha Farhi \\ Earth Observation Department, Centre of Space Techniques, Algeria. \\ * Corresponding author. Tel.: +213 782 441 846; email: \{hmegoufel, hmahi, nfarhi\}@cts.asal.dz \\ Manuscript submitted February 5, 2017; accepted May 9, 2017. \\ doi: 10.17706/ijcee.2017.9.1.343-350
}

\begin{abstract}
In this article, we propose a comparison between several validity indices by combining them with the k-means algorithm in order to determine the optimal number of clusters. The method consists at varying the number of clusters in a predefined range [kmin, kmax] and extracts the best indices values representing the final number of clusters. The compared indices are the Davies-Bouldin index, the Sum of Squares index, the Xie-Benie index, the Artur Starczewski index (SRT), the Pakhira-Bandyopadhyay-Maulik Fuzzy index, the Bayesian information Criterion, the Silhouette index and the Wang \& al index (WSJ). The experimentation and comparison of the validity indices were performed on synthetic and satellite datasets. The results confirm the effectiveness of some indices such as the Sum of Squares index among a large set of data.
\end{abstract}

Key words: Clustering, generation of data, index of validity, K-means.

\section{Introduction}

Clustering is a basic step in image processing. The purpose of this operation is to separate different homogeneous zones of an image, in order to organize the objects into clusters whose members have in common various properties [1]. Clustering methods aim to automatically separate the image into natural clusters without any previous knowledge of the classes. The majority of clustering algorithms suffer from the problem of determining the optimal number of clusters that is often left to the user. To this problem, several measures called validity indices have been implemented to evaluate the quality of the clustering result [2]. Many works combining clustering algorithms and validity indices were carried out. For example, in [3] a study combining the k-Harmonic algorithm and validity indices to determine the optimal number of cluster was proposed.

In this work, we investigate the effectiveness of some indices to determine the optimal number of clusters. The adopted methodology consists at executing the K-means algorithm over a predefined interval [kmin, kmax] then testing the results with the chosen validity indices. Finally, the optimal number of clusters related to the maximum or minimum value of the index is extracted. The rest of the article is organized as follows: the section 2 presents the theoretical aspects relating to both the K-means algorithm and validity indices. The third section is dedicated to the experimentations carried out on both synthetic, composite and satellite data. The last section contains the conclusion.

\section{Methodology}

In this section we give a brief description of the k-means algorithm and the tested clustering validity indices. 


\subsection{K-Means Algorithm}

Defined by McQueen [4], K-means is one of the simplest data classification algorithms. The main idea is to select randomly a set of centers fixed a priori and search iteratively for the optimal partition. Each individual is assigned to the nearest center and after the assignment of all data; the average of each group is calculated to obtain the new representatives of the groups. When the assigned groups reach a stationary state (no data exchanges group) the algorithm is stopped. The K-Means aims at minimizing an objective function that consists in squared error function. This later is defined for a set of observations $(x 1, x 2, \ldots, x n)$, where each observation is a d-dimensional real vector and a set of $k(\leq n)$ partitions $S=\{S 1, S 2, \ldots, S k\}$ as follows:

$$
j=\underset{S}{\arg \min } \sum_{i=1}^{k} \sum_{X \in S_{i}}\|x-\mu\|_{i}^{2}[0,1]
$$

where $\mu_{i}$ is the mean of points in $S_{i}$.

\subsection{Clustering Validity Indices}

In this sub-section, we introduce the clustering validity indices used in this work, namely Davies-Bouldin $(D B)$, Sum of Squares index $(W B)$, Xie-Benie $(X B), S T R$ index $(S T R)$, Pakhira-Bandyopadhyay-Maulik Fuzzy index $(P B M F), B I C$ index $(B I C)$, Silhouette $(S I L)$ index and WSJ index (WSJI)

Davies-Bouldin index (DB): [5] it is a very popular and used crisp index in clustering algorithms. it requires only two parameters to be defined by the user, the distance measure noted $\mathrm{p}$ and the dispersion measure noted $\mathrm{q}$. The $D B$ should be minimize for best clustering and is defined as follows:

$$
D B=\frac{1}{K} \sum_{i=1}^{\mathrm{k}} R_{i}
$$

with

$$
R_{i}=\max _{i, i \neq j}\left\{\frac{S_{i}+S_{j}}{M_{i j}}\right\}
$$

where

$$
S_{i}=\left\{\frac{1}{T_{i}} \sum_{j=1}^{T}\left\|x_{j}-c_{i}\right\|^{q}\right\}^{\frac{1}{q}}
$$

and

$$
M_{i j}=\left\{\sum_{k=1}^{k}\left\|x_{k i}-c_{k j}\right\|^{p}\right\}^{\frac{1}{p}}
$$

with

$c_{k j}: k^{\text {th }}$ Component of the -dimensional vector $C_{j}$.

$c_{i}:$ The center of cluster $i$.

$M_{i j}:$ The Minkowski metric.

$T_{i}:$ The number of vectors (pixels) in clustering.

- Sum of Squares index (WB): [6] it is defined as a ratio of the measure of cluster compactness to its measure of separation. The index is given by:

$$
W B=K \frac{W S S}{S S B}
$$




$$
\begin{array}{r}
\text { WSS }=\sum_{i=1}^{N}\left\|x_{i}-c_{p i}\right\|^{2} \\
S S B=\sum_{i=1}^{\kappa} n_{i}\left\|c_{i}-\bar{X}\right\|^{2}
\end{array}
$$

The lower value of the $W B$ is a strong indicator of good clustering.

- Xie-Benie index (XB): [7] also called $S$ function; $X B$ is defined as a ratio of the total variation to the minimum separation of clusters. The index should be minimized. Its formula is given by:

$$
X B=\frac{1}{N} \frac{\sum_{i=1}^{K} \sum_{j=1}^{N}\left(U_{i j}{ }^{m}\left\|_{x_{j}-c_{i}}\right\|^{2}\right.}{\min _{I \neq}\left\|\left.\right|_{C_{l}-c_{i}}\right\|^{2}}
$$

- STR Index (SRT): [8] introduced by Artur Starczewski, SRT is defined as the product of two components, which determine changes of compactness and separability of clusters during a clustering process. The maximum value of this index identifies the best clustering. Its definition is:

$$
\begin{gathered}
S T R=[E(K)-E(K-1)] \cdot[D(K+1)-D(K)] \\
E(k-1)=\frac{E_{0}}{E_{K-1}} \\
E_{0}=\sum_{x \in X}\|x-c\| \\
E_{K-1}=\sum_{k=1}^{K-1} \sum_{x \in C_{K}}\left\|x-\boldsymbol{C}_{K}\right\| \\
D(K)=\frac{D_{K \max }}{D_{K \min }} \\
D_{K \max }=\max _{i, k=1}\left\|\boldsymbol{C}_{i}-\boldsymbol{c}_{k}\right\| \\
D_{K \min }=\min _{i, k=1}\left\|\boldsymbol{C}_{i}-\boldsymbol{C}_{k}\right\|
\end{gathered}
$$

- WSII Index(WSJI): [9] the index allows a linear combination of averaged fuzzy compactness and separation to measure clustering, which is defined as :

$$
\begin{array}{r}
W S I I(K)=\operatorname{Scat}(K)+\frac{\operatorname{Sep}(K)}{\operatorname{Sep}\left(K_{\max }\right)} \\
\operatorname{Scat}(K)=\frac{\frac{1}{K} \sum_{i=1}^{K}\left\|\sigma\left(z_{i}\right)\right\|}{\|\sigma(X)\|}
\end{array}
$$




$$
\begin{gathered}
\operatorname{Sep}(K)=\frac{D_{\text {max }}^{2}}{D_{\text {min }}} \sum_{i=1}^{k}\left(\sum_{k=1}^{\mathrm{k}}\left\|z_{i}-z_{k}\right\|^{2}\right)^{-1} \\
D_{\text {max }}=\max \left\{\left\|Z_{i^{-}} z_{k}\right\|\right\} \\
D_{\text {min }}=\min \left\{\left\|z_{i}-z_{k}\right\|\right\}
\end{gathered}
$$

The minimum value of the WSJI identifies the best clustering.

- Pakhira-Bandyopadhyay-Maulik Fuzzy index (PBMF): [10] it is considered as a validity index measure for fuzzy clustering. Higher values of the $P B M F$ are preferred. The index is formulated as follows :

$$
P B M F=\left(\begin{array}{l}
1 \\
-\times \frac{E_{1}}{c} J_{m}
\end{array} D_{c}\right)^{2}
$$

with $E 1$ is constant for a given dataset

and

$$
J_{m}^{(U, Z)}=\sum_{j=1}^{n} \sum_{i=1}^{c}\left(m_{i j}\right)^{m}\left\|_{X_{j}-Z_{i}}\right\|
$$

and

$$
D_{c}=\max _{i, j=1}\left\|z_{i}{ }^{-} z_{j}\right\|
$$

- Bayesian information Criterion (BIC) [11]: is a criterion for model selection among a finite set of models; higher values of the $B I C$ are preferred for better clustering. It is based, in part, on the likelihood function and it is formulated as follows:

$$
B I C=\sum_{i=1}^{M}\left(n_{i} \log \frac{n_{i}}{N}-\frac{n_{i} \times D}{2} \log (2 \pi)-\frac{n_{i}}{2} \log \sum_{i}-\frac{n_{i}-M}{2}\right)-\frac{1}{2} M \log N
$$

where

$$
\sum_{i=}=\frac{1}{N-M} \sum_{j=1}^{n_{i}}\|\|_{j}-c_{i} \|^{2}
$$

- Silhouette (SIL) index [8]: The silhouette value is a measure of how similar an object is to its own cluster (cohesion) compared to other clusters (separation). This index can be defined as:

$$
\begin{gathered}
a\left(x_{i}\right)=\frac{1}{n_{m}-1} \sum_{j=1, j \neq i}\left\|_{X_{i}-x_{j}}\right\|_{x_{i} x_{j} \epsilon_{c_{m}}}^{2} \\
b\left(x_{i}\right)=\min \left\{\Sigma_{t \neq m}\|\|_{c_{t}-c_{m}} \|^{2}\right\}_{x_{i} \in \mathcal{C}_{i}} \\
s\left(\boldsymbol{X}_{i}\right)=\frac{b\left(\boldsymbol{X}_{i}\right)-a\left(\boldsymbol{x}_{i}\right)}{\max \left(a\left(\boldsymbol{x}_{i}\right), b\left(\boldsymbol{X}_{i}\right)\right)}
\end{gathered}
$$




$$
S C=\frac{1}{N} \sum_{i=1}^{N} s\left(\mathcal{X}_{i}\right)
$$

The maximum value of the $S I L$ index indicates better clustering.

\section{Experimental Results}

In this part, we propose a comparison of the behavior of the eight tested validity indices on both artificial and real datasets. The second part consists on testing the indices on satellite images acquired by different sensors. The used methodology consists in executing the k-means algorithm over an interval [Kmin, Kmax] with Kmin = 2 and Kmax is given by the rule of thumb [12] [N/2] $]^{0.5}$. The last step consists in determining the optimal number of clusters $\mathrm{K}$ by evaluating the results with the validity indices.

\subsection{Application to Synthetic Data}

In this section, we propose to evaluate and compare the behavior of the cited validity indices. We have chosen a large set of artificial data: S1, S2, S3 and S4 presenting different overlapping rates, a1, a2 and a3 presenting different number of clusters and finally $\operatorname{dim} 032, \operatorname{dim} 064, \operatorname{dim} 128$ and $\operatorname{dim} 256$ with different dimensions. Table 1 describes the characteristics of the data used and the number of clusters returned by the validity indices.

Table 1. Characteristic of the Used Data and Comparison of the Results

\begin{tabular}{|c|c|c|c|c|c|c|c|c|c|c|c|}
\hline \multirow[t]{2}{*}{ Data } & \multirow{2}{*}{$\begin{array}{l}\text { Number } \\
\text { of cluster }\end{array}$} & \multirow[t]{2}{*}{ Dimension } & \multirow{2}{*}{$\begin{array}{l}\text { Point } \\
\text { number }\end{array}$} & \multicolumn{8}{|c|}{ Number of cluster obtained } \\
\hline & & & & $\overline{\mathrm{DB}}$ & WB & $\overline{\mathrm{XB}}$ & "SRT & WSJI & PBMF & BIC & SIL \\
\hline S1 & & & & 14 & $\underline{15}$ & $\underline{15}$ & $\underline{15}$ & $\underline{15}$ & 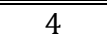 & 50 & $\underline{\underline{15}}$ \\
\hline S2 & & 2 & 5000 & 14 & $\underline{15}$ & 14 & $\underline{15}$ & 13 & 6 & 50 & $\underline{15}$ \\
\hline S3 & 15 & & & 13 & $\underline{15}$ & $\underline{15}$ & 17 & $\underline{15}$ & 5 & 50 & $\underline{15}$ \\
\hline S4 & & & & 16 & $\underline{15}$ & 13 & 16 & 20 & 2 & 50 & $\underline{15}$ \\
\hline a1 & 20 & 2 & 3000 & 19 & $\underline{20}$ & 19 & 23 & 19 & 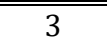 & 39 & 30 \\
\hline a2 & 35 & 2 & 5250 & 32 & 39 & 34 & 38 & 25 & 3 & 50 & 36 \\
\hline a3 & 50 & 2 & 7500 & 43 & 59 & 43 & 44 & 38 & 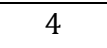 & 61 & 45 \\
\hline dim032 & 16 & 32 & 1024 & 18 & 18 & 15 & 22 & 13 & 7 & 18 & 17 \\
\hline dim064 & 16 & 64 & 1024 & $\underline{16}$ & 17 & 13 & 21 & 14 & 21 & 23 & 18 \\
\hline $\operatorname{dim} 128$ & 16 & 128 & 1024 & 20 & 18 & 13 & 18 & 15 & 18 & 18 & 15 \\
\hline dim256 & 16 & 256 & 1024 & 18 & 17 & 14 & 22 & 14 & 18 & 19 & 18 \\
\hline
\end{tabular}

From Table 1, we can see that the two indices WB and SIL return the exact number of clusters for the S1, S2, S3 and $\mathrm{S} 4$ sets and approximate the optimal value for a2, a3 and dimension data. Moreover, $W B$ returns the exact number of clusters for the a1 dataset. Concerning the $X B, W S J I$ and $R S T$ indices, they often return the exact number of clusters for the S1, S2 and S3 datasets; however, the indices fail at determining the number for the rest of synthetic datasets. We also noticed that the $P B M F$ and $B I C$ indices failed at returning any close values for the majority of the synthetic datasets. Regarding the $D B$ index, the returned values are close to the desired one but lack of precision in comparison with the $W B$ index that gave the better results.

\subsection{Application to Composite Images}

In this section, we propose to compare the behavior of the cited indices of validity on composite data. These later were computed by selecting manually obvious samples from a satellite image. The samples are then resized and concatenated into one final composite image. This way of proceeding allows the construction of synthetic satellite images with known classes in order to perform the indices tests. 


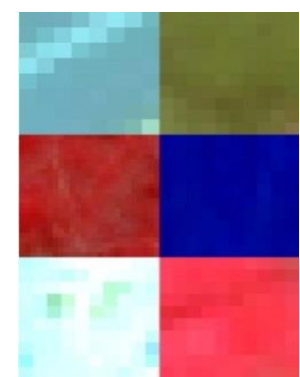

(a)

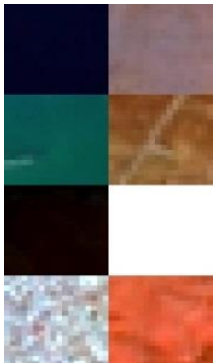

(b)

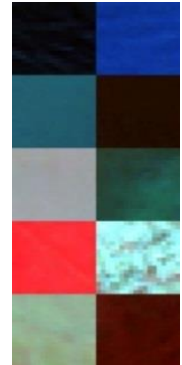

(c)

Fig. 1. Composite images.

Table 2. Results of the Tested Indices on Composite Images

\begin{tabular}{ccccccccc}
\hline $\begin{array}{c}\text { Index } \\
\text { Image }\end{array}$ & DB & WB & XB & SRT & WSJI & PBMF & BIC & SIL \\
\hline \hline (a) & $\underline{\mathbf{6}}$ & $\underline{\mathbf{6}}$ & $\underline{\mathbf{6}}$ & 12 & 2 & 7 & 17 & 8 \\
\hline \hline (b) & 4 & 9 & 5 & 4 & 2 & 4 & 18 & 4 \\
\hline \hline (c) & 8 & 9 & 7 & 4 & 3 & 8 & 2 & 9 \\
\hline \hline
\end{tabular}

Through Table 2, we can see that the $\mathrm{DB}, \mathrm{WB}$ and $\mathrm{XB}$ index return the exact number of cluster for the composite image (a). Regarding the image (b), we notice that the WB index approximates the exact number of cluster overcoming the other validity indices. In contrast, the BIC index gave an erroneous value. For the composite image (c), it is found that the WB and silhouette index approximates the exact value compared to other indices. These results proves again to the adaptability and efficiency of the WB index in comparison with the other tested indices.

\subsection{Application to Satellite Images}

In the last experiment, the clustering has been performed on satellite data by combining the K-Means algorithm with the WB validity index that gave the best testing results. The characteristics of the images set are as follows:

Three Landsat 8 multispectral images, of three different scenes of the Oran region in ALgeria were used: Oran sea in Fig. 2 (a), Oran forest in Fig. 2 (b) and Oran city in Fig. 2 (c). The scenes were aquired with three spectral bands containing $400 \times 400$ pixels over a 30 meters spatial resolution.

The clustering results of the three remotely sensed data obtained by the combination of the K-means and WB are shown in Fig. 2 (d) with eight clusters, Fig. 2 (e) with four clusters and Fig. 2 (f) with twelve clusters, respectively. The visual comparison with the corresponding original images shows that the obtained results appear generally satisfying even if we notice some confusion between water pixels and shadow ones, case of the first image. Regarding the second forest scene, we also notice a lot of confusion between shadows. For the last image, the obtained number of cluster exceeded the desire one, which leads to a lack of visual interpretation of the results.

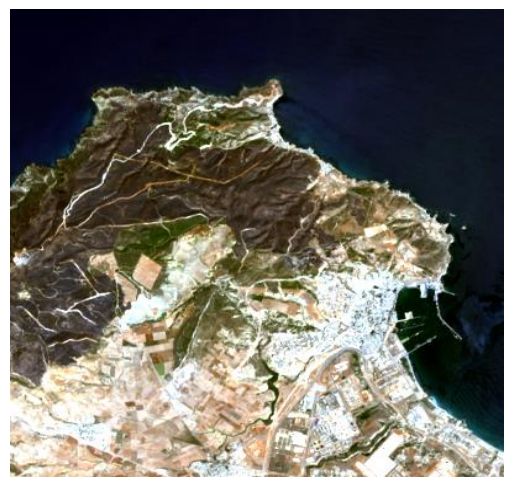

(a)

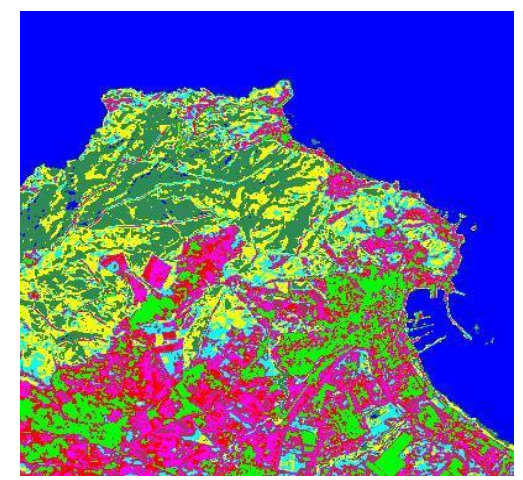

(d) 


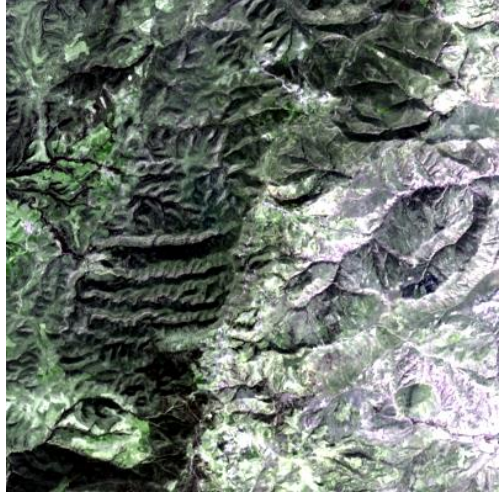

(b)

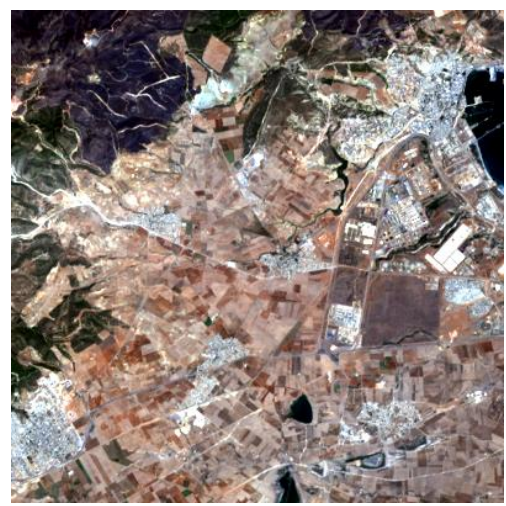

(c)

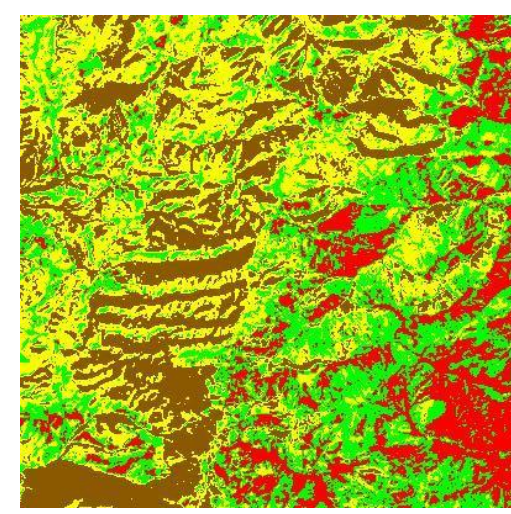

(e)

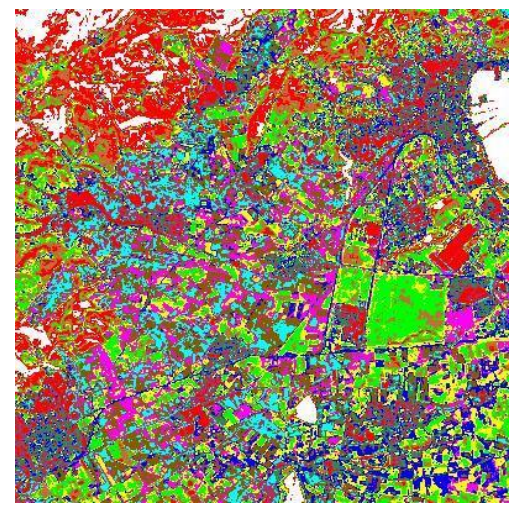

(f)

Fig. 2. Clustering using the k-means and the WB index on satellite data.

\section{Conclusion}

In this article, we performed a comparative study between different validity indices by combining them with the k-means algorithm to extract the optimal number of clusters. The experimentation section was conducted on a large set of synthetic, composite and real data. The obtained results show the effectiveness of the WB index to extract the optimal number of clusters in comparison with the other indices. The tests also demonstrate the adaptability of the tested indices while confronted to different overlapping, dimensions and complexity rates. Further works include the use of the best index in combination with an improved clustering algorithm in order to obtain a new completely automatized clustering method.

\section{References}

[1] Bharill, N., \& Tiwari, A. (July 6-11, 2014). Enhanced Cluster Validity Index for the Evaluation of Optimal Number of Clusters for Fuzzy C-Means Algorithm. Beijing, China.

[2] Arbelaitz, O., Gurrutxaga, I., Muguerza, J., Pérez, M., \& Perona, I. (2013). An extensive comparative study of cluster validity indices. Pattern Recognition, 243-256.

[3] Mahi, H., Farhi, N., \& Labed, K. (2014). Remotely Sensed Data Clustering Using K-Harmonic Means Algorithm and Cluster Validity Index.

[4] McQeen, J. (1967). Some methods for classification and analysis of multivariate observation. Proceedings of $5^{\text {th }}$ Berkeley Symp Mathematics, Statistics and Probability (pp. 281-296).

[5] Davies, D., \& Bouldin, D. (1979). A cluster separation measure. IEEE PAMI, 1, 224-227.

[6] Zhao, Q., \& Fränti, P. (2014). WB Index: A Sum-of-Squares Based Index for Cluster Validity, 77-89.

[7] Xie, X. L., \& Beni, A. (1991). Validity measure for fuzzy clustering. IEEE Trans, 3, 841-846.

[8] Starczewski, A. (2015). A New Validity Index For Crisp Clusters. Poland. 
[9] Sun, H. J., Wang, S. R., \& Jiang, Q. S. (2004). FCM-Based Model Selection Algorithms for Determining the Number of Clusters, 2027-2037.

[10] Pakhira, M. K., Bandyopadhya, S., \& Maulik, U. (2005). A study of some fuzzy cluster validity indices, genetic clustering and application to pixel classification. Fuzzy Sets and Systems, 155, pp. 191-214.

[11] Akaike, (1974). A new look at the statistical model selection identification. IEEE Transaction on Automatic Control, 19, 719-723.

[12] Kodinariya, M., \& Makwana, R. (November 2013). Review on Determining Number of Cluster in K-Means Clustering, 90-95.

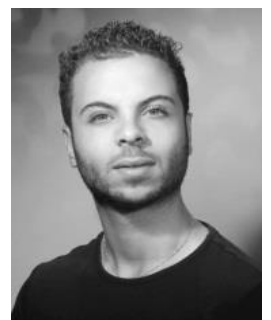

Hanifi Meroufel was born in Oran, Algeria, in 1986. In 2010 he graduated from the Université des Sciences et de la Technologie, Oran, Algérie. In 2012, he received his master in computing specialty recognition in forms and artificial intelligence from the signal, image and parole laboratory (SIMPA). Since 2014, he is a computer engineer at the Centre des Techniques Spatiales, Arzew, Algeria. He belongs to observation of the earth department, and the signal and images processing service. 